\title{
Variational approach for rotating-stellar evolution in Lagrange scheme
}

\author{
Nobutoshi Yasutake ${ }^{1}$ and Shoichi Yamada ${ }^{2}$ \\ ${ }^{1}$ Physics Department, Chiba Institute of Technology, \\ Shibazono 2-1-1, Narashino, Chiba, 275-0023, Japan \\ email: nobutoshi.yasutake@p.chibakoudai.jp \\ ${ }^{2}$ Advanced Research Institute for Science and Engineering, Waseda University, \\ Okubo 3-4-1, Shinjuku, Tokyo, 169-8555, Japan
}

\begin{abstract}
We have developed an entirely new formulation to obtain self-gravitating, axisymmetric configurations in permanent rotation. It is based on the Lagrangian variational principle and, as a consequence, will allow us to apply it to stellar evolution calculations rather easily. We adopt a Monte Carlo technique, which is analogous to those employed in other fields, e.g. nuclear physics, in minimizing the energy functional. We also present the analogies between the study on rotating stellar configurations and the one on deformed nuclei. Possible applications are not limited to main sequence stars but will be extended to e.g. compact stars, proto-stars and planets. We believe that our formulation will be a major break-through then.
\end{abstract}

Keywords. stars: rotation - stars: evolution — stars: protostars

\section{Introduction-Edges and nodes in Lagrange variational principle}

Studies on evolutions of spherically symmetric stars have been advanced exponentially over the years by many researchers (Maeder \& Meynet 2000; Woosley et al. 2002) after the innovation of Henyey method (Henyey et al. 1964). This method is only applicable to spherical (non rotating) topics, and most of the stellar evolution calculations with this method are based on the Lagrange scheme, which is very easy to be applied to stellar evolution calculations. While, in the last ten years, rotation has become an essential part of stellar models comparing with observational results such as Be-stars, there is no appropriate Lagrange scheme for fully multi-dimensional calculations unfortunately.

In this study, we introduce a novel formulation in Lagrange method made of nodes and edges, in which the Lagrange variables, such as mass, angular momentum, entropy, and chemical fractions, are conserved on each node. Our scheme is also based on the variational principle, hence we can get optimal stellar structures finding the minimal energy (N. Yasutake, T. Noda, H. Sotani, T. Maruyama, T. Tatsumi 2013). Although detailed validations will be presented in the sequel, we show an example using our technique.

\section{Deformed-star/pasta (deformed nuclear structures) duality}

We adopt a Monte Carlo technique in variational principle, which is analogous to those employed in other fields, e.g. nuclear physics, in minimizing the energy functional, which is evaluated on a triangulated mesh. We can, actually, find out many analogies between deformed-stars with rotation and deformed nuclear structures; e.g. while deformed nuclear structures, known as "pasta structures", exist under the balance between Coulomb 

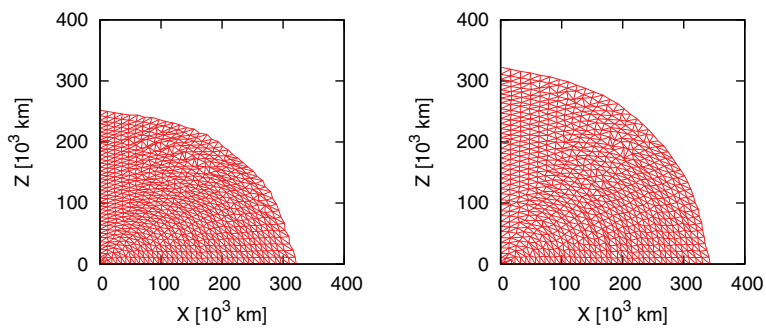

Figure 1. stellar structures in equilibrium shown by edges and nodes. Left panel is the barotropic case, and right one the baroclinic case, where entropy is added to the pole area.

interaction and surface tension (N. Yasutake, T. Noda, H. Sotani, T. Maruyama, T. Tatsumi 2013), the deformed rotating-stars do between gravitation and rotation.

\section{Applicational result and discussion}

In Fig. 1, we show an examples of rotating structures for stellar evolutions. We assume that the stars are composed of hydrogen alone and employ an ideal gas EOS. Left panel shows the barotropic case, where the stellar mass is $0.6 M_{\odot}$, and the entropy is set as $15.0 k_{\mathrm{B}}$ on each node. Here $k_{\mathrm{B}}$ is the Boltzmann's constant. We adopt a spherically symmetric configuration as an initial-guess structure, and add to it the angular momentum, which satisfies the following law on the angular velocity: $\Omega=\Omega_{0} X_{0}^{2} /\left(X^{2}+X_{0}^{2}\right)$. Here $X_{0}$ and $\Omega_{0}$ are model parameters and the former is set to the radius of the spherical star whereas the latter is $\Omega_{0}=10^{-6} \pi \cdot 5000 \mathrm{rad} \mathrm{s}^{-1}$, which is roughly 5000 times the solar angular velocity. Note that the resultant configuration of the angular velocity is given when the initial structure changes to the optimal structure as shown in the left panel.

Right panel shows the baroclinic case, where the entropy has been artificially changed from the barotropic case according to the relation: $K_{i}=K_{0}\left(1+\theta_{i} / \pi\right)$ where $K_{0}$ is the constant of the ideal-gas $\operatorname{EOS} P=K_{0} \rho^{5 / 3}$, and $\theta_{i}$ is the latitude of node $i$.

Note that we can follow up the trajectories of nodes completely; we can see from where to where each node moves in the change of entropy distribution. Although I adopt a simple entropy change here, we can apply realistic nuclear-reactions instead of it. Hence, we can easily apply our technique to stellar evolution calculations. This is one of the strong points on our method.

Another point is that our method is useful to check the instability of stellar structures since our method is based on the variational principle, which is useful for evaluations of mixing regions. We will show it in the nearly future.

We have just assumed a main sequence star here, but our method is applicable to e.g. compact stars, proto-stars and planets. We believe that our formulation will be a major break-through then. We have already checked that our result is consistent with the one by Hachisu method (Hachisu 1986).

\section{References}

Hachisu, I. 1986, ApJS 61, 479

Henyey, L. G., Forbes, J. E., \& Gould, N. L. 1964, ApJ 139, 306

Maeder, A. \& Meynet, G. 2000, ARA\&A 38, 143

N. Yasutake, T. Noda, H. Sotani, T. Maruyama, \& T. Tatsumi 2013, Recent Advances in Quarks Research, Chapt. Thermodynamical description of hadron-quark phase transition and its implications on compact-star phenomena, Nova Publishers

Woosley, S. E., Heger, A., \& Weaver, T. A. 2002, Reviews of Modern Physics 74, 1015 